\title{
Comparative study on the biomechanics between improved PVP and traditional PKP in the treatment of vertebral peripheral wall damage-type OVCF
}

\author{
TAO ZHOU ${ }^{1 *}$, HAO LIN ${ }^{2 *}$, HONGLIANG WANG ${ }^{1}$, XIAOQIANG CHEN $^{1}$ and FANG HE ${ }^{2}$ \\ ${ }^{1}$ Department of Orthopaedics, The People's Hospital of Maanshan; ${ }^{2}$ Clinical College \\ of Maanshan, Anhui Medical University, Maanshan, Anhui 243000, P.R. China
}

Received February 23, 2017; Accepted April 24, 2017

DOI: $10.3892 /$ etm.2017.4542

\begin{abstract}
We compared the biomechanics between improved percutaneous vertebroplasty (improved PVP) and traditional percutaneous kyphoplasty (PKP) in the treatment of vertebral peripheral wall damage-type osteoporotic vertebral compression fracture (OVCF). A total of 15 vertebral peripheral wall damage-type OVCF models of new calves (12-14 weeks) were treated with a decalcifying agent (Shandon TBD-1) with the vertebral compression fracture. The vertebral volume and anterior height before modeling, and the vertebral BMD before and after modeling were measured. The models were randomly divided into three groups: the improved PVP group (Group A), the traditional PKP group (Group B) and the control group (Group C). BMD of Groups A, B and C after decalcification was significantly lower than that before decalcification $(\mathrm{P}<0.05)$. There were no significant differences in BMD before and after decalcification among Groups A, $\mathrm{B}$ and $\mathrm{C}(\mathrm{P}>0.05)$. There was no significant difference in the initial strength and stiffness among the three groups $(\mathrm{P}>0.05)$. The postoperative strength of Groups A and B was 1.036 \pm 300 and $1.045 \pm 200 \mathrm{~N}$, respectively, which was significantly higher than the initial strength $(\mathrm{P}<0.05)$. The postoperative stiffness of Groups A and B was 395 250 and $470 \pm 270 \mathrm{~N} / \mathrm{mm}$, respectively, which was slightly lower than the initial stiffness, however, the differences were not statistically significant
\end{abstract}

Correspondence to: Dr Xiaoqiang Chen, Department of Orthopaedics, The People's Hospital of Maanshan, 45 Hubei Road, Maanshan, Anhui 243000, P.R. China

E-mail: eowm98079280t@163.com

Dr Fang He, Clinical College of Maanshan, Anhui Medical University, 45 Hubei Road, Maanshan, Anhui 243000, P.R. China

E-mail: fanghe001@163.com

${ }^{*}$ Contributed equally

Key words: osteoporotic vertebral compression fracture, peripheral wall damage, improved percutaneous vertebroplasty, traditional percutaneous kyphoplasty, biomechanics
$(\mathrm{P}>0.05)$. In the comparison of postoperative strength and stiffness between Groups A and B, the postoperative strength in Group A was lower than that in Group B; differences were not statistically significant $(\mathrm{P}>0.05)$; there was no significant difference in the postoperative stiffness between Groups A and B $(\mathrm{P}>0.05)$. There were no significant differences of injection of bone cement between the two groups $(\mathrm{P}>0.05)$. Therefore, an improved PVP can basically realize the curative effects of traditional PKP in the treatment of vertebral peripheral wall damage-type OVCF, which can be used as clinical reference.

\section{Introduction}

With the aging population, the occurrence of osteoporotic vertebral compression fracture (OVCF) caused by osteoporosis has been increasing yearly (1). Currently, both traditional PVP and traditional percutaneous kyphoplasty (PKP) can improve clinical symptoms quickly in the treatment of OVCF with good clinical effects (2), and PKP is superior to traditional PVP in correcting kyphosis, recovering the affected vertebra height and reducing the leakage of bone cement, however, PVP is generally used clinically due to the low price, definite curative effect and convenient operation and mastery (3). In the treatment of peripheral wall damage-type OVCF, traditional PVP can cause a leakage of bone cement and other complications (4). The improved percutaneous vertebroplasty (improved PVP) is a type of new technique combined with the advantage and principle of PKP on the basis of traditional PVP. When the bone cement goes into the doughing stage after the drawing stage, it is pushed in using push rod according to the characteristics of permeability and non-permeation of bone cement in the doughing stage in order to realize uniform distribution and expanded support, thus achieving the therapeutic effect of vertebral plasty (5). The comparative study on the biomechanics of improved PVP and traditional PKP can greatly reduce the subjective factors and provide a scientific and stable clinical basis, however, there are few reports in China and other countries due to a lack of effective experimental models. Our study detected a change in two mechanical indexes of vertebral models, compressive strength and stiffness, before and after the improved PVP and traditional PKP, and compared the bone cement filling rate 


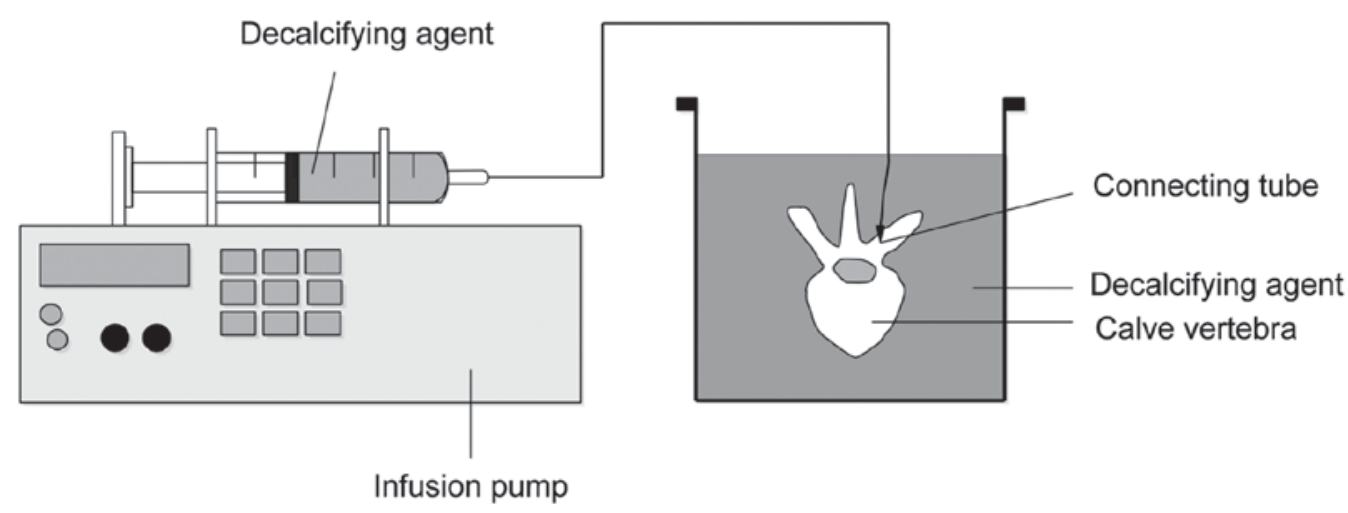

Figure 1. Schematic diagram of decalcification.

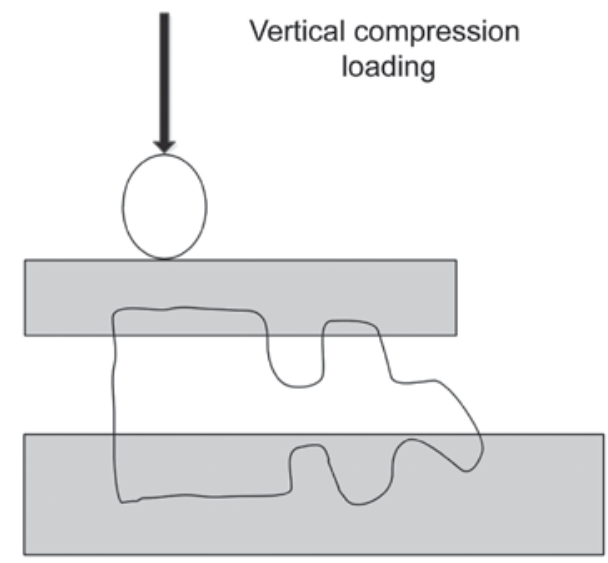

Figure 2. Schematic diagram of compression loading.

between both through vertebral peripheral wall damage-type OVCF models of a new calf (12-14 weeks) treated by decalcifying agent (Shandon TBD-1) with the vertebral compression fracture caused by mechanical device and peripheral wall damage realized by drop weight method (6).

\section{Materials and methods}

Specimens thoracolumbar spine. A total of 15 thoracolumbar vertebra specimens (T9-L4) were taken from 4 new calves (12-14 weeks). Conventional X-ray examination was used to rule out deformity, benign and malignant tumor and fracture, and to remove the surrounding soft tissue immediately and free vertebral body in the intervertebral space. The dual-energy X-ray BMD tester was used to determine the vertebral BMD of each free specimen and the specimen was wrapped by a double-layer plastic wrap and stored in the refrigerator at $-20^{\circ} \mathrm{C}$. The specimen was unfrozen for $24 \mathrm{~h}$ in the refrigerator at $4^{\circ} \mathrm{C}$ before each experiment.

Experimental equipment. Dual-energy X-ray BMD tester (Norland Corp., Fort Atkinson, WI, USA), Micro CT (Scanco Medical AG, Brüttisellen, Switzerland), mechanical test equipment (Department of Mechanics, Anhui University of Technology, Hefei, China), PVP and PKP instrument (Shandong Weigao Group Medical Polymer Co., Ltd., Weihai, China), bone cement (Shandong Weigao Group
Medical Polymer Co., Ltd.), decalcifying agent (Shandon TBD-1) (Thermo Fisher Scientific, Waltham, MA, USA) were all obtained commercially.

Preparation of osteoporosis model. According to literature, we drilled the poles in the intersection of superior articular process periphery and the transverse process on both sides of the vertebral body, respectively (diameter $\mathrm{x}$ depth $=6.5 \times 40 \mathrm{~mm}$ ). We injected $50 \mathrm{ml}$ of decalcifying agent (Shandon TBD-1) into the nail hole on one side of the vertebral body and placed it in a glass bowl filled with decalcifying agent. Then, we used $6 \mathrm{~cm}$-long catheter to connect the infusion pump, and injected $480 \mathrm{ml}$ of the decalcifying agent (Shandon TBD-1), into the nail hole within $12 \mathrm{~h}$ at an average rate of $40 \mathrm{ml} / \mathrm{h}$. The same method was applied to the nail hole on the other side of the vertebral body and each specimen was rinsed by distilled water and dried after decalcification for $24 \mathrm{~h}$. The BMD of specimens before and after decalcification was detected, and the osteoporotic vertebral model was established successfully on the condition that BMD after decalcification was lower than that before decalcification $(\mathrm{P}<0.05$, Fig. 1 for model diagram).

Preparation of vertebral peripheral wall damage-type OVCF. We used the vernier caliper to measure the front and back, left and right height of each vertebral model. We used the dental base acrylic resin powder to embed the upper and lower ends of the vertebral body. We adopted the mechanical test equipment to compress at $5 \mathrm{~mm}$ in posterior margin of the anterior vertebral cortex at a rate of $10 \mathrm{~mm} / \mathrm{min}$ until the vertebral anterior edge was compressed by $25 \%$, thereby achieving the vertebral anterior edge compression fracture; we recorded and drew the strength-displacement curve. According to the knee and slope of the curve, the initial strength and stiffness of each vertebral specimen were obtained. We also used the drop weight method to determine the peripheral wall damage, and measured the front and back, left and right height of each vertebral model, as well as the vertebral volume, again using the vernier caliper. We determined the anterior height of the fractured vertebral body, and used the vertebral CT scan to judge whether there was vertebral peripheral wall damage in order to further determine whether the vertebral peripheral wall damage-type OVCF model of calf was established successfully. This study was approved by the Ethics Committee of the People's Hospital 
Table I. Comparison of basic condition of vertebral models in three groups $(\mathrm{x} \pm \mathrm{s})$.

\begin{tabular}{lccccrc}
\hline Groups & $\begin{array}{c}\text { Sample } \\
\text { size }\end{array}$ & $\begin{array}{c}\text { Vertebral body } \\
\text { volume V2 }(\mathrm{ml})\end{array}$ & $\begin{array}{c}\text { Anterior height } \\
\mathrm{H} 2(\mathrm{~cm})\end{array}$ & $\begin{array}{c}\text { BMD G1 } \\
\left(\mathrm{g} / \mathrm{cm}^{2}\right)\end{array}$ & $\begin{array}{c}\text { BMD G2 } \\
\left(\mathrm{g} / \mathrm{cm}^{2}\right)\end{array}$ & $\begin{array}{c}\text { Bone cement } \\
\mathrm{amount}(\mathrm{ml})\end{array}$ \\
\hline Group A & 5 & $20.03+5.45$ & $2.14 \pm 0.28$ & $1.425 \pm 0.072$ & $1.074 \pm 0.065$ & $3.75 \pm 0.55$ \\
Group B & 5 & $19.76 \pm 7.03$ & $2.20 \pm 0.18$ & $1.482 \pm 0.056$ & $1.059 \pm 0.075$ & $3.65 \pm 0.75$ \\
Group C & 5 & $19.87 \pm 6.65$ & $2.15 \pm 0.25$ & $1.502 \pm 0.063$ & $1.058 \pm 0.081$ & 0.929 \\
$\mathrm{P}_{0}$ & - & 0.847 & 0.414 & 0.885 & 0.478 \\
\hline
\end{tabular}

$\mathrm{P}_{0}>0.05$ without the statistical significance.

Table II. Comparison of statistical results of each index in groups A and B before and after operation.

\begin{tabular}{lccccc}
\hline Group & Initial strength & Postoperative strength & Initial stiffness & Postoperative stiffness & $\mathrm{P}_{2}$ \\
\hline Group A & $700 \pm 220$ & $1.036 \pm 300$ & $450 \pm 230$ & $395 \pm 250$ & 0.021 \\
Group B & $710 \pm 230$ & $1.045 \pm 200$ & $487 \pm 220$ & $470 \pm 270$ & 0.574 \\
$\mathrm{P}_{1}$ & 0.721 & 0.826 & 0.647 & 0.604 & 0.793 \\
\hline
\end{tabular}

$\mathrm{P}_{2}$ and $\mathrm{P}_{3}$ was the comparative result of strength and stiffness in groups $\mathrm{A}$ and $\mathrm{B}$ before and after operation. $\mathrm{P}_{1}$ was the result of pairwise comparison of initial strength, postoperative strength, initial stiffness and postoperative stiffness in groups A and B.

of Maanshan. Signed written informed consents were obtained from all participants before the study.

Vertebral plasty. A total of 15 vertebral models were randomly divided into three groups: the improved PVP group (Group A), traditional PKP group (Group B) and the control group (Group C). The control group was the blank group of peripheral wall damage-type OVCF model, namely the group without processing. There was no significant difference in the vertebral volume, BMD and initial stiffness and strength of the vertebral body among the three groups. The operation process was generally the same as that in literature (3), and the key to the improved PVP was that the bone cement in the doughing stage was pushed in using a push rod when the bone cement came into the doughing stage after drawing stage. The vertebral anterior height of both groups was measured.

Biomechanical experiment. After the injection of bone cement, all vertebral bodies were stored in plastic bags and placed in the refrigerator at $4^{\circ} \mathrm{C}$ for $24 \mathrm{~h}$; they were retrieved for the experiment. The dental base acrylic resin powder was used to fix the single vertebral body, and then the anterior edge of bone cement-enhanced vertebral body was compressed at the rate of $10 \mathrm{~mm} / \mathrm{min}$ until it was compressed by $25 \%$ in order to measure the stiffness and strength (Fig. 2).

Imaging observation. Micro CT was conducted for specimens in three groups in order to ensure the formation of vertebral peripheral wall damage-type OVCF model, and Micro CT was conducted again after the vertebral plasty was used to observe the bone cement filling rate.

Data collection. The following data were collected from specimens: i) BMD of each vertebral body before and after decalcification; ii) the front and back, left and right height of the vertebral body before and after the model building; iii) the anterior height of the fractured vertebral body after model building and operations in Groups A and B; iv) initial intensity and initial stiffness after model building; strength and stiffness of the vertebral body after operations in Groups A and B and v) bone cement filling rate.

Statistical processing. SPSS 13.0 software (SPSS, Inc., Chicago, IL, USA) was used, and the paired t-test was used in the comparison of BMD, strength and stiffness, and BMD, initial strength and initial stiffness before decalcification in each group. The ANOVA test was used in the intergroup comparison and $\mathrm{P}<0.05$ suggested that the difference was statistically significant.

\section{Results}

Basic condition of vertebral body. During the whole test process, the vertebral body of the calf did not suffer from comminuted fracture, and all of the 15 vertebral models that were selected were anterior wall damage with a similar damage degree. BMD after decalcification in Groups A, $\mathrm{B}$ and $\mathrm{C}$ was significantly lower than that before decalcification $(\mathrm{P}<0.05)$, and there was no significant difference in BMD before and after decalcification among the three groups $(\mathrm{P}<0.05)$. After the establishment of vertebral models and grouping, there were no statistically significant differences in the anterior height and vertebral body volume among the three groups $(\mathrm{P}>0.05)$. The bone cement in both improved PVP and traditional PKP exceeded the middle line of vertebral body, and the difference was not statistically significant in the bone cement filling amount between Groups A and B (P>0.05, Table I). 
Comparison of strength and stiffness values in Groups A and $B$ before and after operation. The postoperative strength in Groups A and B after the operation was 1.036 \pm 300 and $1.045 \pm 200$, respectively, which was significantly higher than the initial strength in both groups $(\mathrm{P}<0.05)$. The postoperative stiffness in Groups A and B after the operation was $395 \pm 250$ and $470 \pm 270 \mathrm{~N} / \mathrm{mm}$, respectively, which was slightly lower than initial stiffness, however, the differences were not statistically significant $(\mathrm{P}>0.05)$. In the comparison of postoperative strength and stiffness in Groups A and B, the postoperative strength in Group A was lower than that in Group B; differences were not statistically significant $(\mathrm{P}>0.05)$. There was no significant difference in postoperative stiffness between Groups A and B (P>0.05, Table II).

\section{Discussion}

Overview of minimally invasive therapy of OVCF. With regards to the surgical treatment of OVCF, vertebral plasty, as an emerging minimally invasive spinal technique, can rapidly ease pain, promote restoration of affected vertebral height, and is characterized by a simple operation, short learning curve and high safety, which can shorten the patient's bed-rest time, reduce related complications, and significantly improve quality of life (7). In terms of the clinical effects, traditional PVP and PKP, as two types of traditional vertebral plasty, can significantly alleviate a patients' pain without significant difference (8). However, the PKP is superior to traditional PVP in correcting kyphosis and recovering the vertebral height since the difference of PKP lies in that the special inflatable balloon that is injected into the vertebral body through the working channel before the injection of bone cement. The high pressure injector is connected to expand the balloon in order to form a larger cavity, though the core of the two types of surgical methods is to inject bone cement into the vertebral body. Therefore, the former is uniform dispersion, while the latter is the non-uniform dispersion. In addition, the injection amount of bone cement in PKP is more than that in traditional PVP, which greatly enhances the stiffness and strength of the vertebral body, and therefore, the PKP is superior to traditional PVP in correcting the kyphosis and recovering vertebral height (9). In addition, the balloon expansion reduces the incidence of bone cement leakage and other surgical complications, thus, most scholars tend to choose the traditional PKP for clinical treatment of peripheral wall damage-type OVCF and traditional PVP can cause leakage of bone cement and other complications (4). Of course, traditional PVP is characterized by a simple operation, definite curative effect and low cost, and therefore, it is widely used in treatment of general-type OVCF.

Mechanism of treatment of OVCF with improved PVP. The improved PVP is a type of new technique that is combined with the advantage and principle of PKP on the basis of traditional PVP. With the help of the full understanding of the performance of bone cement, when the bone cement comes into the doughing stage after the drawing stage, it is pushed in using a push rod according to the characteristics of permeability and non-permeation of bone cement in doughing stage, which fully combines the characteristics of uniform distribu- tion of bone cement in traditional PVP (10). This avoids the disadvantages of traditional PVP of bone cement leakage with the similar effects of balloon dilatation support to PKP (11). An improved PVP can determine the treatment effects of vertebral plasty, and reduce the risk of complications, such as permeation.

Possibility, selection and method of establishing effective experimental vertebral animal model quickly. There is a paucity of research on the clinical effects of improved PVP and traditional PKP on the peripheral wall damage-type OVCF, and there are fewer reports in China and other countries due to a lack of effective biomechanics in the experimental model (12). Therefore, the establishment of animal model of peripheral wall damage-type OVCF is the key to this study. According to literature, the diagnostic standard of osteoporosis in BMD is 2.5 times lower than the mean peak of BMD of normal bones (13), and the mechanical characteristics that constitute the bones are generally considered as a combination of collagen fibers and calcium phosphate, among which calcium phosphate determines the bone stiffness (14), and the content of calcium is closely related to vertebral BMD and bone strength (15), therefore, decalcification can cause vertebral osteoporosis. Research has shown that the reason behind vertebral fracture is that the stress exceeds the strength of the trabecular bone, and the main structure of vertebral body is able to bear, and the trabecular bone structure is damaged and the local fracture is further developed, which leads to the vertebral fracture (16). In conclusion, if decalcification can be realized rapidly and vertebral compression fracture with peripheral wall damage can be achieved by external force, then the peripheral wall damage-type OVCF animal model will be established successfully.

There are many osteoporosis animal models tht are currently used in spinal biomechanics research, which are represented by castrated female animals or medicine-taking animals (17). It is the living animal model, and is considered to be the most satisfactory model for evaluating the efficiency of a chemical agent and investigating the pathophysiological mechanism of osteoporosis (18); however, it has the disadvantages of time-consuming modeling, complex operation and vulnerability to external environment interference (12), and therefore, it is not suitable for the biomechanical experiments. Now, the most commonly-used model for evaluating biological materials and efficiency of spinal internal fixation repair system is the new and ripe calf spinal specimen (19). Combined with a large number of study reports, the author used a vertebral peripheral wall damage-type OVCF model of a new calf (12-14 weeks) that was treated with a decalcifying agent (Shandon TBD-1) with the vertebral compression fracture caused by mechanical device and peripheral wall damage that was determined by the drop weight method for the experiment. The experimental results showed that after being decalcified using Shandon TBD-1, the change in vertebral BMD was obvious with significant differences, and the vertebral osteoporotic change was achieved completely, which was characterized by a shorter processing time, less external disturbance and simple operation; it was also safer than nitric acid and other decalcifying agents. Moreover, the compression fracture was caused by the $25 \%$ compression of anterior verte- 
bral body with the knee and slope of force-displacement curve, which was in accordance with the experimental demand. The height was changed similarly and the multi-factor influence was reduced.

Comparison ofbiomechanics of improved PVP and traditional $P K P$ in treatment of vertebral peripheral wall damage-type $O V C F$. The anterior wall damage degree of experimental models in this study are similar, and the bone cement in both the improved PVP and traditional PKP exceeds the middle line of the vertebral body, which indirectly indicates the full dispersion of both kinds of operations. Bone cement has little impact on the mechanical equilibrium on either side of the vertebral body, and the injection of bone cement can enhance the strength and stiffness of the whole vertebral body. The bone cement filling rate was similar in the process of experiments without statistical difference, and therefore, there was no case of bone cement leakage. The bone cement injection speed and method were the same in the whole vertebral plasty, which shows that the safety factor of improved PVP was higher than that of traditional PVP, which might be associated with the bone cement status in the doughing stage, or the similar effect of bone cement to balloon dilatation support in doughing stage.

It is reported in the literature that there is a close correlation between the stiffness and strength of bone (20-22), and the detection of vertebral compressive strength and stiffness can help evaluate the biomechanical properties of vertebral body or implants (23-25). This experiment detects the change in the two mechanical indexes of vertebral models, compressive strength and stiffness, before and after improved PVP and traditional PKP. The comparison of compressive strength before and after the two kinds of operations showed that the bone cement injection could quickly restore and enhance the strength of injured vertebra, and the comparison of postoperative strength after the two types of operations indicated that the strength of injured vertebra after improved PVP was lower than that after traditional PKP; the difference was not statistically significant $(\mathrm{P}>0.05)$, suggesting that the improved PVP can determine the strength of injured vertebra similar to traditional PKP. The comparison of preoperative stiffness before and after the two types of operations showed that the injection of bone cement could not fully recover the stiffness of injured vertebra. The stiffness was reduced compared to that of the normal osteoporotic vertebral body, and the difference was not statistically significant, which was considered to be caused by the vertebral compression-induced vertebral cortex damage (6). The comparison of postoperative stiffness after two types of operations, indicated that the difference in postoperative stiffness was not statistically significant after improved PVP and traditional PKP $(\mathrm{P}>0.05)$. Overall, improved PVP can provide sufficient biomechanical strength and stiffness and meet the needs of injured vertebra.

In conclusion, in terms of biomechanics, improved PVP can basically achieve the curative effect of traditional PKP in the treatment of vertebral peripheral wall damage-type $\mathrm{OVCF}$, however, considering the non-human specimen and the limited number of calf specimens in this study, it has certain limitation and therefore, our results must be used for reference only.

\section{References}

1. Melton LJ, Kallmes DF: Epidemiology of vertebral fractures: Implications for vertebral augmentation. Acad Radio1 13: 538-545, 2006.

2. Oldenhuis CN, Oosting SF, Gietema JA and de Vries EG: Prognostic versus predictive value of biomarkers in oncology. Eur J Cancer 44: 946-953, 2008.

3. Tseng YY, Lo YL, Chen LH, Lai PL and Yang ST: Percutaneous polymethylmethacrylate vertebroplasty in the treatment of pain induced by metastatic spine tumor. Surg Neurol 70 (Suppl 1): S78-S83, 2008.

4. Taylor RS, Taylor RJ and Fritzell P: Balloon kyphoplasty and vertebroplasty for vertebral compression fractures: A comparative systematic review of efficacy and safety. Spine 31: 2747-2755, 2006

5. Azorit C, Hervas J, Analla M, Carrasco R and Muñoz-Cobo J: Histological thin-sections: A method for the microscopic study of teeth in Spanish red deer (Cervus elaphus hispanicus). Anat Histol Embryol 31: 224-227, 2002.

6. Tomita S, Kin A, Yazu M and Abe M: Biomechanical evaluation of kyphoplasty and vertebroplasty with calcium phosphate cement in a simulated osteoporotic compression fracture. J Orthop Sci 8: 192-197, 2003.

7. Boonen S, Wahl DA, Nauroy L, Brandi ML, Bouxsein ML, Goldhahn J, Lewiecki EM, Lyritis GP, Marsh D, Obrant K, et al; CSA Fracture Working Group of International Osteoporosis Foundation: Balloon kyphoplasty and vertebroplasty in the management of vertebral compression fractures. Osteoporos Int 22: 2915-2934, 2011.

8. Watts NB, Harris ST and Genant HK: Treatment of painful osteoporotic vertebral fractures with percutaneous vertebroplasty or kyphoplasty. Osteoporos Int 12: 429-437, 2001.

9. Mathis JM: Percutaneous vertebroplasty or kyphoplasty: Which one do I choose? Skeletal Radiol 35: 629-631, 2006.

10. Lamy O, Uebelhart B and Aubry-Rozier B: Risks and benefits of percutaneous vertebroplasty or kyphoplasty in the management of osteoporotic vertebral fractures. Osteoporos Int 25: 807-819, 2014.

11. Zhao G, Liu X and Li F: Balloon kyphoplasty versus percutaneous vertebroplasty for treatment of osteoporotic vertebral compression fractures (OVCFs). Osteoporos Int 27: 2823-2834, 2016.

12. Anselmetti GC, Muto M, Guglielmi G and Masala S: Percutaneous vertebroplasty or kyphoplasty. Radiol Clin North Am 48: 641-649, 2010

13. Seven A, Yuksel B, Kabil Kucur S, Yavuz G, Polat M, Unlu BS and Keskin N: The evaluation of hormonal and psychological parameters that affect bone mineral density in postmenopausal women. Eur Rev Med Pharmacol Sci 20: 20-25, 2016.

14. Cho AR, Kim HK, Kwon JY, Kim TK, Choi YM and Kim KH: The incorporation of platelet-rich plasma into calcium phosphate cement enhances bone regeneration in osteoporosis. Pain Physician 17: E737-E745, 2014.

15. Gao J, Mi S and Liu C: Treatment of various kinds of osteoporotic vertebral fracture with vertebral plasty. Chin J Bone Jt Inj 24: 35-37, 2009 (In Chinese).

16. Colangelo D, Nasto LA, Genitiempo M, Formica VM, Autore G, Pambianco V, Tamburrelli FC, Cerulli G and Pola E: Kyphoplasty vs conservative treatment: a case-control study in 110 post-menopausal women population. Is kyphoplasty better than conservative treatment? Eur Rev Med Pharmacol Sci 19: 3998-4003, 2015

17. Zarrinkalam MR, Beard H, Schultz CG and Moore RJ: Validation of the sheep as a large animal model for the study of vertebral osteoporosis. Eur Spine J 18: 244-253, 2009.

18. Nayak S, Olkin I, Liu H, Grabe M, Gould MK, Allen IE, Owens DK and Bravata DM: Meta-analysis: Accuracy of quantitative ultrasound for identifying patients with osteoporosis. Ann Intern Med 144: 832-841, 2006.

19. Egermann M, Goldhahn J and Schneider E: Animal models for fracture treatment in osteoporosis. Osteoporos Int 16 (Suppl 2): S129-S138, 2005.

20. Nakagi Y, Ito T, Hirooka K, Sugioka Y, Endo H, Saijo Y, Imai H, Takeda H, Kayama F, Sasaki S, et al: Association between lifestyle habits and bone mineral density in Japanese juveniles. Environ Health Prev Med 15: 222-228, 2010. 
21. Kilincer C, Inceoglu S, Sohn MJ, Ferrara LA, Bakirci N, Benzel EC: Load sharing within a human thoracic vertebral body: An in vitro biomechanical study. Turk Neurosurg 17: 167-177, 2007.

22. Giro G, Gonçalves D, Sakakura CE, Pereira RM, Marcantonio Júnior E and Orrico SR: Influence of estrogen deficiency and its treatment with alendronate and estrogen on bone density around osseointegrated implants: Radiographic study in female rats. Oral Surg Oral Med Oral Pathol Oral Radiol Endod 105: 162-167, 2008.

23. Shirke SS, Jadhav SR, Jagtap AG: Methanolic extract of Cuminum cyminum inhibits ovariectomy induced bone loss in rats. Exp Biol Med 233: 1403-1410, 2008.
24. Martin-Monge E, Tresguerres IF, Blanco L, Khraisat A, Rodríguez-Torres R and Tresguerres JA: Validation of an osteoporotic animal model for dental implant analyses: An in vivo densitometric study in rabbits. Int J Oral Maxillofac Implants 26: 725-730, 2011.

25. Fyhrie DP and Vashishth D: Bone stiffness predicts strength similarly for human vertebral cancellous bone in compression and for cortical bone in tension. Bone 26: 169-173, 2000. 